\title{
The puzzles of dark matter searches
}

\author{
Maxim Yu. Khlopov \\ APC laboratory 10, rue Alice Domon et Léonie Duquet \\ 75205 Paris Cedex 13, France \\ Centre for Cosmoparticle Physics "Cosmion" 115409 Moscow, Russia; \\ Moscow Engineering Physics Institute (National Nuclear Research University), 115409 Moscow, Russia
}

\begin{abstract}
Positive results of dark matter searches in DAMA/NaI and DAMA/LIBRA experiments, being put together with negative results of other groups, can imply nontrivial particle physics solutions for cosmological dark matter. Stable particles with charge -2 bind with primordial helium in O-helium "atoms" $(\mathrm{OHe})$, representing a specific Warmer than Cold nuclearinteracting form of dark matter. Slowed down in the terrestrial matter, OHe is elusive for direct methods of underground Dark matter detection like those used in CDMS experiment, but its reactions with nuclei can lead to annual variations of energy release in the interval of energy $2-6 \mathrm{keV}$ in DAMA/NaI and DAMA/LIBRA experiments. Schrodinger equation for system of nucleus and $\mathrm{OHe}$ is considered and reduced to an equation of relative motion in a spherically symmetrical potential well, formed by the Yukawa tail of nuclear scalar isoscalar attraction potential, acting on He beyond the nucleus, and dipole Coulomb repulsion between the nucleus and $\mathrm{OHe}$ at distances from the nuclear surface, smaller than the size of $\mathrm{OHe}$. The values of coupling strength and mass of meson, mediating scalar isoscalar nuclear potential, are rather uncertain. Within these uncertainties we find a narrow window of these parameters, at which the sodium and/or iodine nuclei have a few keV binding energy with $\mathrm{OHe}$. The concentration of $\mathrm{OHe}$ in the matter of underground detectors is adjusted to the incoming flux of cosmic O-helium at the timescale less than few minutes. Therefore the rate of radiative capture of $\mathrm{Na}$ and/or I by $\mathrm{OHe}$ should experience annual modulations. Transitions to more energetic levels of $\mathrm{Na}+\mathrm{OHe}(\mathrm{I}+\mathrm{OHe})$ system imply tunneling through dipole Coulomb barrier that leads to suppression of annual modulation of events with MeV-tens MeV energy release in the correspondence with the results of DAMA experiments. The proposed explanation inevitably leads to prediction of abundance of anomalous $\mathrm{Na}$ (and/or I) corresponding to the signal, observed by DAMA. At nuclear parameters, reproducing DAMA results, the energy release predicted for detectors with chemical content other than NaI differ in the most cases from the one in DAMA detector. In particular, it is shown that in the case of CDMS the energy of OHe-germanium bound state is beyond the range of 2-6 keV and its formation should not lead to ionization in the energy interval of DAMA signal.
\end{abstract}

Keywords: elementary particles, nuclear reactions, nucleosynthesis, abundances, dark matter, early universe, large-scale structure of universe PACS: $12.60 . \mathrm{Cn}, 98.90 .+\mathrm{s}, 12.60 . \mathrm{Nz}, 14.60 . \mathrm{Hi}, 26.35 .+\mathrm{c}, 36.90 .+\mathrm{f}, 03.65 . \mathrm{Ge}$

\section{INTRODUCTION}

The widely shared belief is that the dark matter, corresponding to $25 \%$ of the total cosmological density, is nonbaryonic and consists of new stable particles. One can formulate the set of conditions under which new particles can be considered as candidates to dark matter (see e.g. [1, 2, 3] for review and reference): they should be stable, saturate the measured dark matter density and decouple from plasma and radiation at least before the beginning of matter dominated stage. The easiest way to satisfy these conditions is to involve neutral weakly interacting particles. However it is not the only particle physics solution for the dark matter problem. In the composite dark matter scenarios new stable particles can have electric charge, but escape experimental discovery, because they are hidden in atom-like states maintaining dark matter of the modern Universe.

It offers new solutions for the physical nature of the cosmological dark matter. The main problem for these solutions is to suppress the abundance of positively charged species bound with ordinary electrons, which behave as anomalous isotopes of hydrogen or helium. This problem is unresolvable, if the model predicts stable particles with charge -1 , as it is the case for tera-electrons [4, 5]. To avoid anomalous isotopes overproduction, stable particles with charge -1 should be absent, so that stable negatively charged particles should have charge -2 only.

Elementary particle frames for heavy stable -2 charged species are provided by: (a) stable "antibaryons" $\bar{U} \bar{U} \bar{U}$ formed by anti- $U$ quark of fourth generation [6, 7, 8, 9] (b) AC-leptons [9, 10, 11], predicted in the extension [10] of standard model, based on the approach of almost-commutative geometry [12]. (c) Technileptons and antitechnibaryons [13] in the framework of walking technicolor models (WTC) [14]. (d) Finally, stable charged clusters $\bar{u}_{5} \bar{u}_{5} \bar{u}_{5}$ of (anti)quarks $\bar{u}_{5}$ of 5 th family can follow from the approach, unifying spins and charges [15]. 
In the asymmetric case, corresponding to excess of -2 charge species, $X^{--}$, as it was assumed for $(\bar{U} \bar{U} \bar{U})^{--}$in the model of stable $U$-quark of a 4th generation, as well as can take place for $\left(\bar{u}_{5} \bar{u}_{5} \bar{u}_{5}\right)^{--}$in the approach [15] their positively charged partners effectively annihilate in the early Universe. Such an asymmetric case was realized in [13] in the framework of WTC, where it was possible to relate the excess of negatively charged anti-techni-baryons $(\bar{U} \bar{U})^{--}$ and/or technileptons $\zeta^{--}$to the baryon asymmetry of the Universe. The relationship between baryon asymmetry and excess of -2 charge stable species is supported by sphaleron transitions at high temperatures and can be realized in all the models, in which new stable species belong to non-trivial representations of electroweak SU(2) group.

After it is formed in the Standard Big Bang Nucleosynthesis (SBBN), ${ }^{4} \mathrm{He}$ screens the $X^{--}$charged particles in composite $\left({ }^{4} \mathrm{He}^{++} \mathrm{X}^{--}\right)$O-helium "atoms" [7]. For different models of $\mathrm{X}^{--}$these "atoms" are also called ANOhelium [8, 9], Ole-helium [9, 11] or techni-O-helium [13]. We'll call them all O-helium $(\mathrm{OHe})$ in our further discussion, which follows the guidelines of [16].

In all these forms of O-helium, $X^{--}$behaves either as lepton or as specific "heavy quark cluster" with strongly suppressed hadronic interaction. Therefore O-helium interaction with matter is determined by nuclear interaction of $H e$. These neutral primordial nuclear interacting objects contribute to the modern dark matter density and play the role of a nontrivial form of strongly interacting dark matter [17, 18]. The active influence of this type of dark matter on nuclear transformations seems to be incompatible with the expected dark matter properties. However, it turns out that the considered scenario is not easily ruled out [7, 11, 13, 19] and challenges the experimental search for various forms of O-helium and its charged constituents.

Here after a brief review of main features of OHe Universe we concentrate on its effects in underground detectors. We present following [20] a qualitative confirmation of the earlier guess [7, 16, 21] that the positive results of dark matter searches in DAMA/NaI (see for review [22]) and DAMA/LIBRA [23] experiments can be explained by effect of O-helium, resolving the controversy between these data and negative results of other experimental groups.

\section{O-HELIUM UNIVERSE}

Following [7, 8, 9, 13, 16] consider charge asymmetric case, when excess of $X^{--}$provides effective suppression of positively charged species.

In the period $100 \mathrm{~s} \leq t \leq 300 \mathrm{~s}$ at $100 \mathrm{keV} \geq T \geq T_{o}=I_{o} / 27 \approx 60 \mathrm{keV},{ }^{4} \mathrm{He}$ has already been formed in the SBBN and virtually all free $X^{--}$are trapped by ${ }^{4} \mathrm{He}$ in O-helium "atoms" $\left({ }^{4} \mathrm{He}^{++} \mathrm{X}^{--}\right)$. Here the O-helium ionization potential is]

$$
I_{o}=Z_{x}^{2} Z_{H e}^{2} \alpha^{2} m_{H e} / 2 \approx 1.6 \mathrm{MeV}
$$

where $\alpha$ is the fine structure constant, $Z_{H e}=2$ and $Z_{x}=2$ stands for the absolute value of electric charge of $X^{--}$. The size of these "atoms" is [7, 11]

$$
R_{o} \sim 1 /\left(Z_{x} Z_{H e} \alpha m_{H e}\right) \approx 2 \cdot 10^{-13} \mathrm{~cm}
$$

Here and further, if not specified otherwise, we use the system of units $\hbar=c=k=1$.

O-helium, being an $\alpha$-particle with screened electric charge, can catalyze nuclear transformations, which can influence primordial light element abundance and cause primordial heavy element formation. These effects need a special detailed and complicated study. The arguments of [7, 11, 13] indicate that this model does not lead to immediate contradictions with the observational data.

Due to nuclear interactions of its helium constituent with nuclei in the cosmic plasma, the O-helium gas is in thermal equilibrium with plasma and radiation on the Radiation Dominance (RD) stage, while the energy and momentum transfer from plasma is effective. The radiation pressure acting on the plasma is then transferred to density fluctuations of the O-helium gas and transforms them in acoustic waves at scales up to the size of the horizon.

At temperature $T<T_{o d} \approx 200 S_{3}^{2 / 3} \mathrm{eV}$ the energy and momentum transfer from baryons to O-helium is not effective [7, 13] because

$$
n_{B}\langle\sigma v\rangle\left(m_{p} / m_{o}\right) t<1,
$$

where $m_{o}$ is the mass of the $\mathrm{OHe}$ atom and $S_{3}=m_{o} /(1 \mathrm{TeV})$. Here

$$
\sigma \approx \sigma_{o} \sim \pi R_{o}^{2} \approx 10^{-25} \mathrm{~cm}^{2},
$$

\footnotetext{
${ }^{1}$ The account for charge distribution in $H e$ nucleus leads to smaller value $I_{o} \approx 1.3 \mathrm{MeV}[24]$.
} 
and $v=\sqrt{2 T / m_{p}}$ is the baryon thermal velocity. Then O-helium gas decouples from plasma. It starts to dominate in the Universe after $t \sim 10^{12} \mathrm{~s}$ at $T \leq T_{R M} \approx 1 \mathrm{eV}$ and O-helium "atoms" play the main dynamical role in the development of gravitational instability, triggering the large scale structure formation. The composite nature of O-helium determines the specifics of the corresponding dark matter scenario.

At $T>T_{R M}$ the total mass of the $O H e$ gas with density $\rho_{d}=\left(T_{R M} / T\right) \rho_{t o t}$ is equal to

$$
M=\frac{4 \pi}{3} \rho_{d} t^{3}=\frac{4 \pi}{3} \frac{T_{R M}}{T} m_{P l}\left(\frac{m_{P l}}{T}\right)^{2}
$$

within the cosmological horizon $l_{h}=t$. In the period of decoupling $T=T_{o d}$, this mass depends strongly on the Ohelium mass $S_{3}$ and is given by [13]

$$
M_{o d}=\frac{T_{R M}}{T_{o d}} m_{P l}\left(\frac{m_{P l}}{T_{o d}}\right)^{2} \approx 2 \cdot 10^{44} S_{3}^{-2} \mathrm{~g}=10^{11} S_{3}^{-2} M_{\odot},
$$

where $M_{\odot}$ is the solar mass. O-helium is formed only at $T_{o}$ and its total mass within the cosmological horizon in the period of its creation is $M_{o}=M_{o d}\left(T_{o d} / T_{o}\right)^{3}=10^{37} \mathrm{~g}$.

On the RD stage before decoupling, the Jeans length $\lambda_{J}$ of the $\mathrm{OHe}$ gas was restricted from below by the propagation of sound waves in plasma with a relativistic equation of state $p=\varepsilon / 3$, being of the order of the cosmological horizon and equal to $\lambda_{J}=l_{h} / \sqrt{3}=t / \sqrt{3}$. After decoupling at $T=T_{o d}$, it falls down to $\lambda_{J} \sim v_{o} t$, where $v_{o}=\sqrt{2 T_{o d} / m_{o}}$. Though after decoupling the Jeans mass in the $\mathrm{OHe}$ gas correspondingly falls down

$$
M_{J} \sim v_{o}^{3} M_{o d} \sim 3 \cdot 10^{-14} M_{o d},
$$

one should expect a strong suppression of fluctuations on scales $M<M_{o}$, as well as adiabatic damping of sound waves in the RD plasma for scales $M_{o}<M<M_{o d}$. It can provide some suppression of small scale structure in the considered model for all reasonable masses of O-helium. The significance of this suppression and its effect on the structure formation needs a special study in detailed numerical simulations. In any case, it can not be as strong as the free streaming suppression in ordinary Warm Dark Matter (WDM) scenarios, but one can expect that qualitatively we deal with Warmer Than Cold Dark Matter model.

Being decoupled from baryonic matter, the $\mathrm{OHe}$ gas does not follow the formation of baryonic astrophysical objects (stars, planets, molecular clouds...) and forms dark matter halos of galaxies. It can be easily seen that O-helium gas is collisionless for its number density, saturating galactic dark matter. Taking the average density of baryonic matter one can also find that the Galaxy as a whole is transparent for O-helium in spite of its nuclear interaction. Only individual baryonic objects like stars and planets are opaque for it.

\section{SIGNATURES OF O-HELIUM DARK MATTER}

The composite nature of O-helium dark matter results in a number of observable effects.

\section{Anomalous component of cosmic rays}

O-helium atoms can be destroyed in astrophysical processes, giving rise to acceleration of free $X^{--}$in the Galaxy.

O-helium can be ionized due to nuclear interaction with cosmic rays [7, 16]. Estimations [7, 25] show that for the number density of cosmic rays $n_{C R}=10^{-9} \mathrm{~cm}^{-3}$ during the age of Galaxy a fraction of about $10^{-6}$ of total amount of OHe is disrupted irreversibly, since the inverse effect of recombination of free $X^{--}$is negligible. Near the Solar system it leads to concentration of free $X^{--} n_{X}=3 \cdot 10^{-10} S_{3}^{-1} \mathrm{~cm}^{-3}$. After OHe destruction free $X^{--}$ have momentum of order $p_{X} \cong \sqrt{2 \cdot M_{X} \cdot I_{o}} \cong 2 \mathrm{GeVS}_{3}^{1 / 2}$ and velocity $v / c \cong 2 \cdot 10^{-3} S_{3}^{-1 / 2}$ and due to effect of Solar modulation these particles initially can hardly reach Earth [21, 25]. Their acceleration by Fermi mechanism or by the collective acceleration forms power spectrum of $X^{--}$component at the level of $X / p \sim n_{X} / n_{g}=3 \cdot 10^{-10} S_{3}^{-1}$, where $n_{g} \sim 1 \mathrm{~cm}^{-3}$ is the density of baryonic matter gas.

At the stage of red supergiant stars have the size $\sim 10^{15} \mathrm{~cm}$ and during the period of this stage $\sim 3 \cdot 10^{15} \mathrm{~s}$, up to $\sim 10^{-9} S_{3}^{-1}$ of O-helium atoms per nucleon can be captured [21, 25]. In the Supernova explosion these OHe atoms are 
disrupted in collisions with particles in the front of shock wave and acceleration of free $X^{--}$by regular mechanism gives the corresponding fraction in cosmic rays.

If these mechanisms of $X^{--}$acceleration are effective, the anomalous low $Z / A$ component of -2 charged $X^{--}$can be present in cosmic rays at the level $X / p \sim n_{X} / n_{g} \sim 10^{-9} S_{3}^{-1}$, and be within the reach for PAMELA and AMS02 cosmic ray experiments.

In the framework of Walking Tachnicolor model the excess of both stable $X^{--}$and $Y^{++}$is possible [21], the latter being two-three orders of magnitude smaller, than the former. It leads to the two-component composite dark matter scenario with the dominant OHe accompanied by a subdominant WIMP-like component of $\left(X^{--} Y^{++}\right)$bound systems. Technibaryons and technileptons can be metastable and decays of $X^{--}$and $Y^{++}$can provide explanation for anomalies, observed in high energy cosmic positron spectrum by PAMELA and in high energy electron spectrum by FERMI and ATIC.

\section{Positron annihilation and gamma lines in galactic bulge}

Inelastic interaction of O-helium with the matter in the interstellar space and its de-excitation can give rise to radiation in the range from few $\mathrm{keV}$ to few $\mathrm{MeV}$. In the galactic bulge with radius $r_{b} \sim 1 \mathrm{kpc}$ the number density of O-helium can reach the value $n_{o} \approx 3 \cdot 10^{-3} / S_{3} \mathrm{~cm}^{-3}$ and the collision rate of O-helium in this central region was estimated in [16]: $d N / d t=n_{o}^{2} \sigma v_{h} 4 \pi r_{b}^{3} / 3 \approx 3 \cdot 10^{42} S_{3}^{-2} \mathrm{~s}^{-1}$. At the velocity of $v_{h} \sim 3 \cdot 10^{7} \mathrm{~cm} / \mathrm{s}$ energy transfer in such collisions is $\Delta E \sim 1 \mathrm{MeVS}_{3}$. These collisions can lead to excitation of O-helium. If $2 \mathrm{~S}$ level is excited, pair production dominates over two-photon channel in the de-excitation by $E 0$ transition and positron production with the rate $3 \cdot 10^{42} S_{3}^{-2} \mathrm{~s}^{-1}$ is not accompanied by strong gamma signal. According to [26] this rate of positron production for $S_{3} \sim 1$ is sufficient to explain the excess in positron annihilation line from bulge, measured by INTEGRAL (see [27 for review and references). If $\mathrm{OHe}$ levels with nonzero orbital momentum are excited, gamma lines should be observed from transitions $(n>m) E_{n m}=1.598 \mathrm{MeV}\left(1 / m^{2}-1 / n^{2}\right)$ (or from the similar transitions corresponding to the case $\left.I_{o}=1.287 \mathrm{MeV}\right)$ at the level $3 \cdot 10^{-4} S_{3}^{-2}\left(\mathrm{~cm}^{2} \mathrm{~s} \mathrm{MeVster}\right)^{-1}$.

\section{O-HELIUM IN THE TERRESTRIAL MATTER}

The evident consequence of the O-helium dark matter is its inevitable presence in the terrestrial matter, which appears opaque to O-helium and stores all its in-falling flux.

After they fall down terrestrial surface the in-falling $\mathrm{OHe}$ particles are effectively slowed down due to elastic collisions with matter. Then they drift, sinking down towards the center of the Earth with velocity

$$
V=\frac{g}{n \sigma v} \approx 80 S_{3} A^{1 / 2} \mathrm{~cm} / \mathrm{s} .
$$

Here $A \sim 30$ is the average atomic weight in terrestrial surface matter, $n=2.4 \cdot 10^{24} / A$ is the number of terrestrial atomic nuclei, $\sigma v$ is the rate of nuclear collisions and $g=980 \mathrm{~cm} / \mathrm{s}^{2}$.

Near the Earth's surface, the O-helium abundance is determined by the equilibrium between the in-falling and down-drifting fluxes.

The in-falling O-helium flux from dark matter halo is

$$
F=\frac{n_{0}}{8 \pi} \cdot\left|\overline{V_{h}}+\overline{V_{E}}\right|
$$

where $V_{h}$-speed of Solar System $(220 \mathrm{~km} / \mathrm{s}), V_{E}$-speed of Earth $(29.5 \mathrm{~km} / \mathrm{s})$ and $n_{0}=3 \cdot 10^{-4} S_{3}^{-1} \mathrm{~cm}^{-3}$ is the $10 \mathrm{cal}$ density of O-helium dark matter. Here, for qualitative estimation, we don't take into account velocity dispersion and distribution of particles in the incoming flux that can lead to significant effect.

At a depth $L$ below the Earth's surface, the drift timescale is $t_{d r} \sim L / V$, where $V \sim 400 S_{3} \mathrm{~cm} / \mathrm{s}$ is given by Eq. (5). It means that the change of the incoming flux, caused by the motion of the Earth along its orbit, should lead at the depth $L \sim 10^{5} \mathrm{~cm}$ to the corresponding change in the equilibrium underground concentration of $\mathrm{OHe}$ on the timescale $t_{d r} \approx 2.5 \cdot 10^{2} S_{3}^{-1} \mathrm{~s}$. by

The equilibrium concentration, which is established in the matter of underground detectors at this timescale, is given

$$
n_{o E}=\frac{2 \pi \cdot F}{V}=n_{0} \frac{n \sigma v}{4 g} \cdot\left|\overline{V_{h}}+\overline{V_{E}}\right|,
$$


where, with account for $V_{h}>V_{E}$, relative velocity can be expressed as

$$
\begin{gathered}
\left|\overline{V_{o}}\right|=\sqrt{\left(\overline{V_{h}}+\overline{V_{E}}\right)^{2}}=\sqrt{V_{h}^{2}+V_{E}^{2}+V_{h} V_{E} \sin (\theta)} \simeq \\
\simeq V_{h} \sqrt{1+\frac{V_{E}}{V_{h}} \sin (\theta)} \sim V_{h}\left(1+\frac{1}{2} \frac{V_{E}}{V_{h}} \sin (\theta)\right) .
\end{gathered}
$$

Here $\theta=\omega\left(t-t_{0}\right)$ with $\omega=2 \pi / T, T=1 y r$ and $t_{0}$ is the phase. Then the concentration takes the form

$$
n_{o E}=n_{o E}^{(1)}+n_{o E}^{(2)} \cdot \sin \left(\omega\left(t-t_{0}\right)\right)
$$

So, there are two parts of the signal: constant and annual modulation, as it is expected in the strategy of dark matter search in DAMA experiment [23].

Such neutral $\left({ }^{4} \mathrm{He}^{++} \mathrm{X}^{--}\right)$"atoms" may provide a catalysis of cold nuclear reactions in ordinary matter (much more effectively than muon catalysis). This effect needs a special and thorough investigation. On the other hand, $X^{--}$ capture by nuclei, heavier than helium, can lead to production of anomalous isotopes, but the arguments, presented in $[7,11,13]$ indicate that their abundance should be below the experimental upper limits.

It should be noted that the nuclear cross section of the O-helium interaction with matter escapes the severe constraints [18] on strongly interacting dark matter particles (SIMPs) [17, 18] imposed by the XQC experiment [28]. Therefore, a special strategy of direct O-helium search is needed, as it was proposed in [29].

In underground detectors, $\mathrm{OHe}$ "atoms" are slowed down to thermal energies and give rise to energy transfer $\sim 2.5 \cdot 10^{-4} \mathrm{eVA} / S_{3}$, far below the threshold for direct dark matter detection. It makes this form of dark matter insensitive to the severe CDMS constraints [30]. However, in $\mathrm{OHe}$ reactions with the matter of underground detectors can lead to observable effects. Following earlier guess [7, 16, 21, 31] it was shown in [20] that such reactions in NaI

can explain the results of DAMA/NaI and DAMA/LIBRA experiments.

\section{LOW ENERGY BOUND STATE OF O-HELIUM WITH NUCLEI}

The explanation [20] is based on the idea that OHe, slowed down in the matter of DAMA/NaI or DAMA/LIBRA detector, can form a few $\mathrm{keV}$ bound state with nucleus, in which $\mathrm{OHe}$ is situated beyond the nucleus. Therefore the positive result of this experiment is explained by reaction

$$
A+\left({ }^{4} H e^{++} X^{--}\right) \rightarrow\left[A\left({ }^{4} H e^{++} X^{--}\right)\right]+\gamma
$$

with sodium and/or iodine. In detectors with different chemical content such level may not exist at all, or has other value of energy, making the the comparison with DAMA results a nontrivial task.

\section{Low energy bound state of O-helium with nuclei}

The approach of [20] assumes the following picture: at the distances larger, than its size, OHe is neutral and it feels only Yukawa exponential tail of nuclear attraction, due to scalar-isoscalar nuclear potential. It should be noted that scalar-isoscalar nature of He nucleus excludes its nuclear interaction due to $\pi$ or $\rho$ meson exchange, so that the main role in its nuclear interaction outside the nucleus plays $\sigma$ meson exchange, on which nuclear physics data are not very definite. When the distance from the surface of nucleus becomes smaller than the size of OHe, the mutual attraction of nucleus and $\mathrm{OHe}$ is changed by dipole Coulomb repulsion. Inside the nucleus strong nuclear attraction takes place. In the result the spherically symmetric potential appears, given by

$$
U=-\frac{A_{H e} A g^{2} \exp (-\mu r)}{r}+\frac{Z_{H e} Z e^{2} r_{o} \cdot F(r)}{r^{2}} .
$$

Here $A_{H e}=4, Z_{H e}=2$ are atomic weight and charge of helium, $A$ and $Z$ are respectively atomic weight and charge of nucleus, $\mu$ and $g^{2}$ are the mass and coupling of scalar-isoscalar meson - mediator of nuclear attraction, $r_{o}$ is the size of $\mathrm{OHe}$ and $F(r)$ is its electromagnetic formfactor, which strongly suppresses the strength of dipole electromagnetic interaction outside the OHe "atom". 


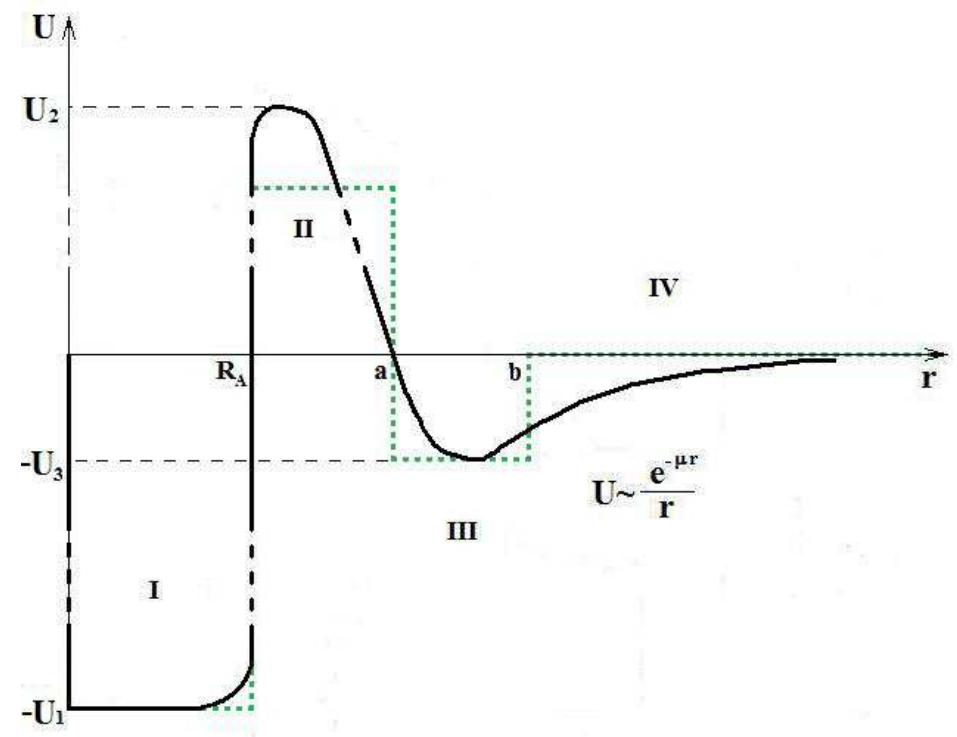

FIGURE 1. The approximation of rectangular well for potential of OHe-nucleus system.

Schrodinger equation for this system is reduced (taking apart the equation for the center of mass) to the equation of relative motion for the reduced mass.

In the case of orbital momentum $l=0$ the wave functions depend only on $r$.

To simplify the solution of Schrodinger equation the potential (96) was approximated in [20] by a rectangular potential that consists of a deep potential well within the radius of nucleus $R_{A}$, of a rectangular dipole Coulomb potential barrier outside its surface up to the radial layer $a=R_{A}+r_{o}$, where it is suppressed by the OHe atom formfactor, and of the outer potential well of the width $\sim 1 / \mu$, formed by the tail of Yukawa nuclear interaction. It leads to the approximate potential [20], presented on Fig. 1]

Solutions of Schrodinger equation for each of the four regions, indicated on Fig. 1, are given in textbooks (see e.g. [32]) and their sewing determines the condition, under which a low-energy OHe-nucleus bound state appears in the region III.

The energy of this bound state and its existence strongly depend on the parameters $\mu$ and $g^{2}$ of nuclear potential (9). On the Fig. 2 the region of these parameters, giving 2-6 keV energy level in OHe bound states with sodium and iodine are presented. In these calculations [20] the mass of $\mathrm{OHe}$ was taken equal to $m_{o}=1 \mathrm{TeV}$.

The rate of radiative capture of $\mathrm{OHe}$ by nuclei should be accurately calculated with the use of exact form of wave functions, obtained for the OHe-nucleus bound state. This work is now in progress. One can use the analogy with the radiative capture of neutron by proton with the following corrections:

- There is only E1 transition in the case of OHe capture.

- The reduced masses of n-p and OHe-nucleus systems are different

- The existence of dipole Coulomb barrier leads to a suppression of the cross section of OHe radiative capture.

With the account for these effects our first estimations give the rate of OHe radiative capture, reproducing the level of signal, detected by DAMA.

Formation of OHe-nucleus bound system leads to energy release of its binding energy, detected as ionization signal in DAMA experiment. In the context of the approach [20] the existence of annual modulations of this signal in the range 2-6 keV and absence of such effect at energies above $6 \mathrm{keV}$ means that binding energy of Na-OHe and I-OHe systems should not exceed $6 \mathrm{keV}$, being in the range $2-6 \mathrm{keV}$ for at least one of these elements. These conditions were taken into account for determination of nuclear parameters, at which the result of DAMA can be reproduced. At these values of $\mu$ and $g^{2}$ energy of OHe binding with other nuclei can strongly differ from 2-6 keV. In particular, energy release at the formation of OHe bound state with thallium can be larger than $6 \mathrm{keV}$. However, assuming $A^{-2}$ dependence for the cross section of radiative capture of nuclei by $\mathrm{OHe}$ and taking into account that thallium content in DAMA detector is 3 orders of magnitude smaller, than NaI, such signal is to be below the experimental errors. 


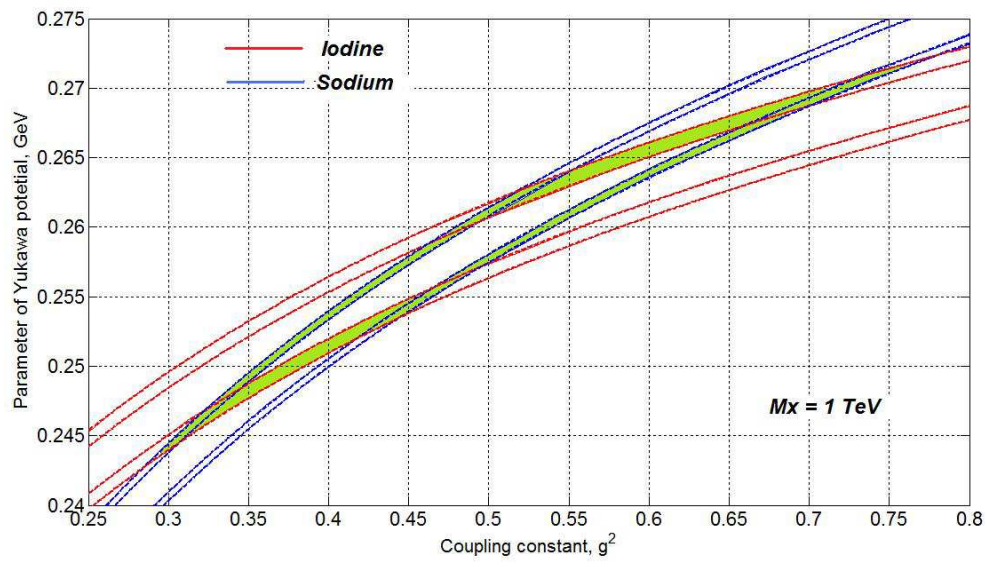

FIGURE 2. The region of parameters $\mu$ and $g^{2}$, for which $\mathrm{Na}$ and I have a level in the interval 2-6 keV. For each nucleus two narrow strips determine the region of parameters, at which the bound system of this element with OHe has a level in 2-6 keV energy range. The outer line of strip corresponds to the level of $6 \mathrm{keV}$ and the internal line to the level of $2 \mathrm{keV}$. The region of intersection of strips correspond to existence of 2-6 keV levels in both OHe-Na and OHe-I systems, while the piece of strip between strips of other nucleus corresponds to the case, when OHe bound state with this nucleus has 2-6 keV level, while the binding energy of OHe with the other nuclei is less than $2 \mathrm{keV}$ by absolute value.

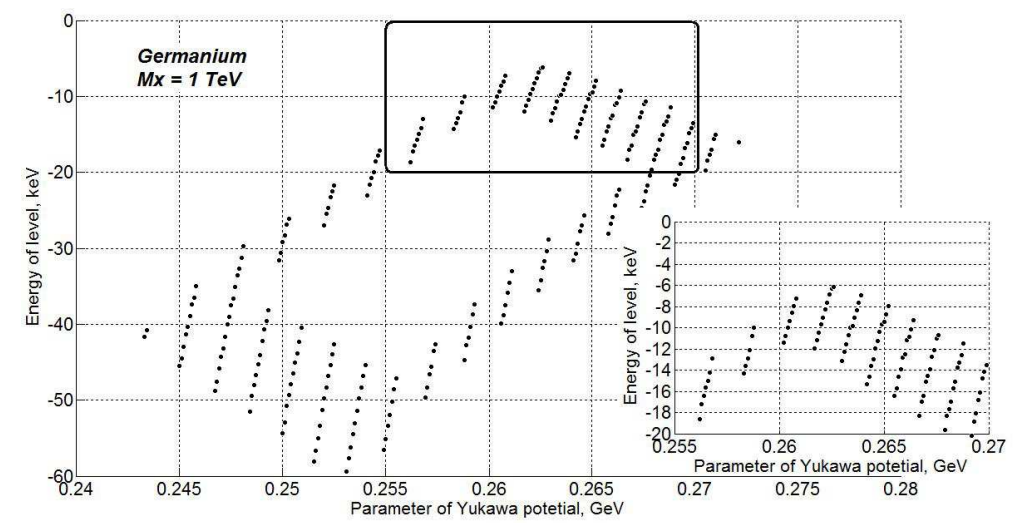

FIGURE 3. Energy levels in OHe bound system with germanium. The range of energies close to energy release in DAMA experiment is blown up to demonstrate that even in this range there is no formal intersection with DAMA results.

It should be noted that the results of DAMA experiment exhibit also absence of annual modulations at the energy of MeV-tens MeV. Energy release in this range should take place, if OHe-nucleus system comes to the deep level inside the nucleus (in the region I of Fig. (1). This transition implies tunneling through dipole Coulomb barrier and is suppressed below the experimental limits.

\section{Energy levels in other nuclei}

For the chosen range of nuclear parameters, reproducing the results of DAMA/NaI and DAMA/LIBRA, the binding energy of OHe-nucleus states in nuclei, corresponding to chemical composition of set-ups in other experiments were calculated in [20]. The results of such calculation for germanium, corresponding to the CDMS detector of experiment, are presented on Fig. 3. For all the parameters, reproducing results of DAMA experiment the predicted energy level of OHe-germanium bound state is beyond the range $2-6 \mathrm{keV}$, being dominantly in the range of tens - few-tens keV by absolute value. It makes elusive a possibility to test DAMA results by search for ionization signal in the same range 2-6 keV in other set-ups with content that differs from $\mathrm{Na}$ and I. In particular, our approach naturally predicts absence 


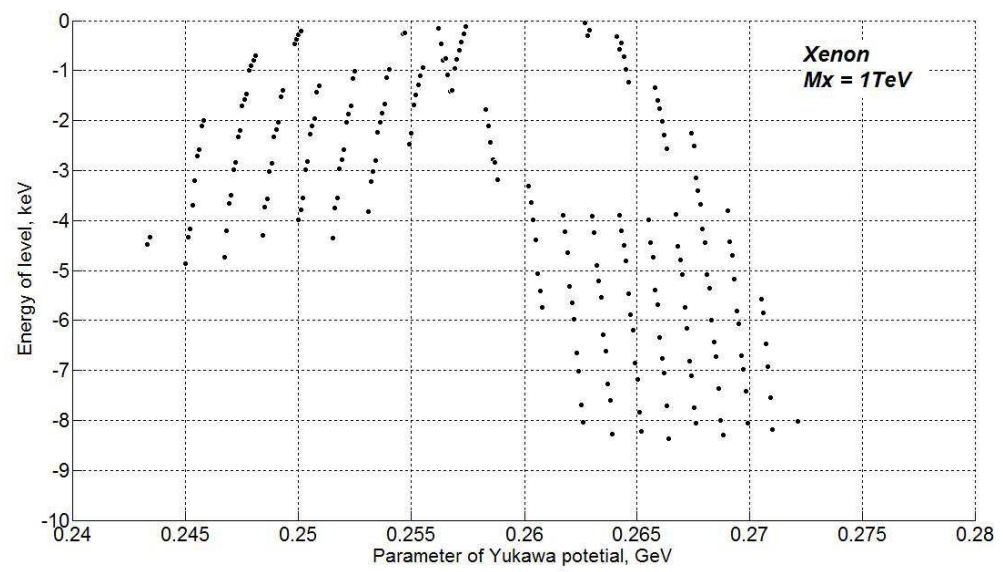

FIGURE 4. Energy levels in OHe bound system with xenon.

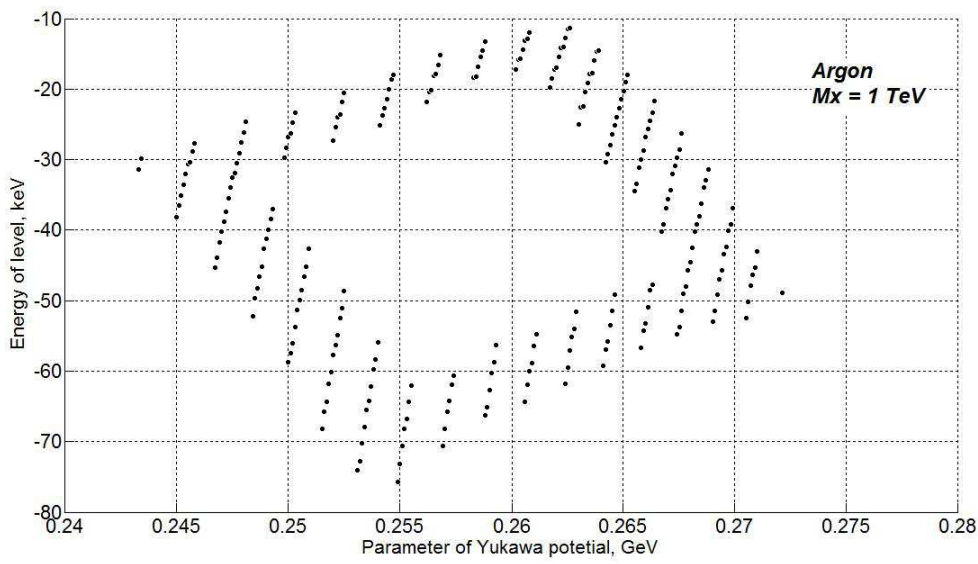

FIGURE 5. Energy levels in OHe bound system with argon.

of ionization signal in the range $2-6 \mathrm{keV}$ in accordance with the recent results of CDMS [33]. 6).

There were also calculated the energies of bound states of OHe with xenon (Fig. 4), argon (Fig. 5) and carbon (Fig.

With the growth of the mass of O-helium the reduced mass of OHe-nucleus system slightly grows, approaching with higher accuracy the mass of nucleus. It extends a bit the range of nuclear parameters $\mu$ and $g^{2}$, at which the binding energy of OHe with sodium and/or iodine is within the range 2-6 keV. Qualitatively, the predictions for superheavy O-helium are similar to the case of $S_{3}=1$.

\section{CONCLUSIONS}

To conclude, the existence of heavy stable charged particles may not only be compatible with the experimental constraints but even lead to composite dark matter scenario of nuclear interacting Warmer than Cold Dark Matter. This new form of dark matter can provide explanation of excess of positron annihilation line radiation, observed by INTEGRAL in the galactic bulge. The search for stable -2 charge component of cosmic rays is challenging for PAMELA and AMS02 experiments. Decays of heavy charged constituents of composite dark matter can provide explanation for anomalies in spectra of cosmic high energy positrons and electrons, observed by PAMELA, FERMI and ATIC. In the context of our approach search for heavy stable charged quarks and leptons at LHC acquires the significance of experimental probe for components of cosmological composite dark matter. 


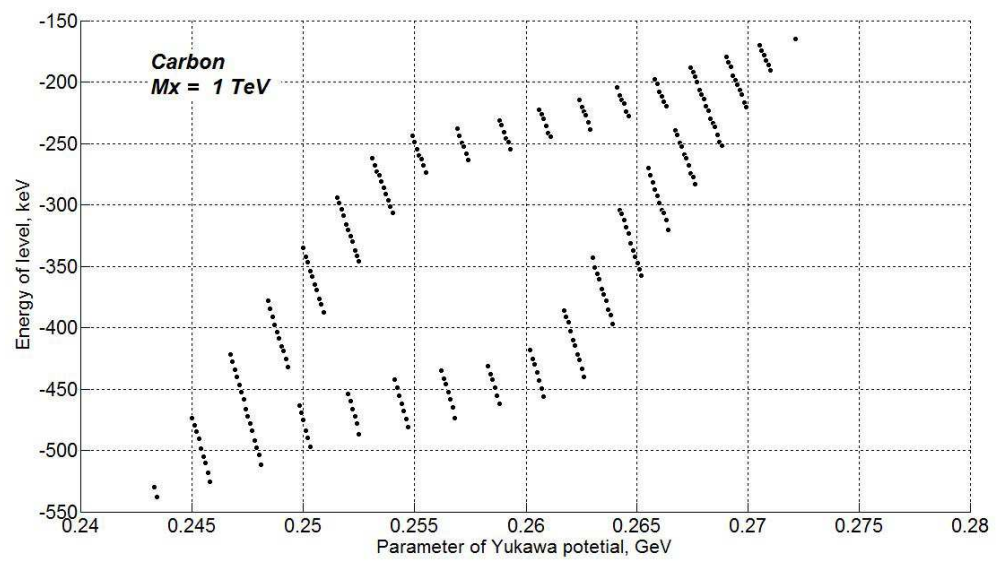

FIGURE 6. Energy levels in OHe bound system with carbon.

The results of dark matter search in experiments DAMA/NaI and DAMA/LIBRA can be explained in the framework of our scenario without contradiction with negative results of other groups. This scenario can be realized in different frameworks, in particular, in the extensions of Standard Model, based on the approach of almost commutative geometry, in the model of stable quarks of 4th generation that can be naturally embedded in the heterotic superstring phenomenology, in the models of stable technileptons and/or techniquarks, following from Minimal Walking Technicolor model or in the approach unifying spin and charges. Our approach contains distinct features, by which the present explanation can be distinguished from other recent approaches to this problem [34] (see also review and more references in [35] as well as in the corresponding contributions to the present Proceedings).

The proposed explanation is based on the mechanism of low energy binding of OHe with nuclei. Within the uncertainty of nuclear physics parameters there exists a range at which $\mathrm{OHe}$ binding energy with sodium and/or iodine is in the interval 2-6 keV. Radiative capture of $\mathrm{OHe}$ to this bound state leads to the corresponding energy release observed as an ionization signal in DAMA detector.

OHe concentration in the matter of underground detectors is determined by the equilibrium between the incoming cosmic flux of $\mathrm{OHe}$ and diffusion towards the center of Earth. It is rapidly adjusted and follows the change in this flux with the relaxation time of few minutes. Therefore the rate of radiative capture of OHe should experience annual modulations reflected in annula modulations of the ionization signal from these reactions.

An inevitable consequence of the proposed explanation is appearance in the matter of DAMA/NaI or DAMA/LIBRA detector anomalous superheavy isotopes of sodium and/or iodine, having the mass roughly by $m_{o}$ larger, than ordinary isotopes of these elements. If the atoms of these anomalous isotopes are not completely ionized, their mobility is determined by atomic cross sections and becomes about 9 orders of magnitude smaller, than for O-helium. It provides their conservation in the matter of detector. Therefore mass-spectroscopic analysis of this matter can provide additional test for the O-helium nature of DAMA signal. Methods of such analysis should take into account the fragile nature of $\mathrm{OHe}-\mathrm{Na}$ bound states, since their binding energy is only few keV.

With the account for high sensitivity of the numerical results to the values of nuclear parameters and for the approximations, made in the calculations, the presented results can be considered only as an illustration of the possibility to explain puzzles of dark matter search in the framework of composite dark matter scenario. An interesting feature of this explanation is a conclusion that the ionization signal expected in detectors with the content, different from NaI, can be dominantly in the energy range beyond 2-6 keV. Therefore test of results of DAMA/NaI and DAMA/LIBRA experiments by other experimental groups can become a very nontrivial task.

Our results show that the ionization signal, detected by DAMA, may be absent in detectors containing light elements. In particular, there is predicted no low-energy binding of $\mathrm{OHe}$ with ${ }^{3} \mathrm{He}$ and correspondingly no ionization signal in $\mathrm{keV}$ range in the designed ${ }^{3} \mathrm{He}$ dark matter detectors. Therefore development of experimental methods of dark matter detection will extend the possibilities to test hypothesis of composite dark matter.

The presented approach sheds new light on the physical nature of dark matter. Specific properties of composite dark matter and its constituents are challenging for their experimental search. OHe interaction with matter is an important aspect of these studies. In this context positive result of DAMA/NaI and DAMA/LIBRA experiments may be a signature for exciting phenomena of O-helium nuclear physics. 


\section{ACKNOWLEDGMENTS}

I express my gratitude to K.M. Belotsky, C. Kouvaris, A.G. Mayorov and E. Yu. Soldatov for collaboration in obtaining the presented results and to Jean Pierre Gazeau for discussions.

\section{REFERENCES}

1. M.Yu. Khlopov Cosmoparticle physics (World Scientific, Singapore, 1999).

2. M.Yu. Khlopov in Cosmion-94, Eds. M.Yu.Khlopov et al. (Editions frontieres, 1996) P. 67; M. Y. Khlopov in hep-ph/0612250 , p 51.

3. M. Y. Khlopov, Bled Workshops in Physics 8, 114 (2007); in arXiv:0711.4681, p. 114; M. Y. Khlopov and N. S. MankocBorstnik, ibid, p. 195.

4. S. L. Glashow, arXiv:hep-ph/0504287.

5. D. Fargion and M. Khlopov, arXiv:hep-ph/0507087

6. K.M.Belotsky et al, Gravitation and Cosmology 11, 3 (2005)

7. M.Yu. Khlopov, JETP Lett. 83, 1 (2006).

8. K. Belotsky et al, arXiv:astro-ph/0602261 K. Belotsky et al, Gravitation and Cosmology 12, 1 (2006); K. Belotsky et al, arXiv:0806.1067 [astro-ph].

9. M. Y. Khlopov, arXiv:astro-ph/0607048

10. C. A. Stephan, arXiv:hep-th/0509213

11. D. Fargion et al, Class. Quantum Grav. 23, 7305 (2006); M. Y. Khlopov and C. A. Stephan, arXiv:astro-ph/0603187

12. A. Connes Noncommutative Geometry (Academic Press, London and San Diego, 1994).

13. M. Y. Khlopov and C. Kouvaris, Phys. Rev. D 77, 065002 (2008).

14. F. Sannino and K. Tuominen, Phys. Rev. D 71, 051901 (2005); D. K. Hong et al, Phys. Lett. B 597, 89 (2004); D. D. Dietrich et al, Phys. Rev. D 72, 055001 (2005); D. D. Dietrich et al, arXiv:hep-ph/0510217, S. B. Gudnason et al, Phys. Rev. D 73, 115003 (2006); S. B. Gudnason et al, Phys. Rev. D 74, 095008 (2006).

15. N.S. Mankoč Borštnik, This Volume; A. Borštnik Bračič, N.S. Mankoč Borštnik, Phys. Rev. D 74, 073013 (2006); N.S. Mankoč Borštnik, Mod. Phys. Lett. A 10, 587 (1995); N.S. Mankoč Borštnik, Int. J. Theor. Phys. 40, 315 (2001); G. Bregar, M. Breskvar, D. Lukman, N.S. Mankoč Borštnik, New J. of Phys. 10, 093002 (2008).

16. M. Y. Khlopov, arXiv:0806.3581 [astro-ph].

17. C. B. Dover et al, Phys. Rev. Lett. 42, 1117 (1979); S. Wolfram, Phys. Lett. B 82, 65 (1979); G. D. Starkman et al, Phys. Rev. D 41, 3594 (1990); D. Javorsek et al, Phys. Rev. Lett. 87, 231804 (2001); S. Mitra, Phys. Rev. D 70, 103517 (2004); G. D. Mack et al, Phys. Rev. D 76, 043523 (2007);

18. B. D. Wandelt et al., arXiv:astro-ph/0006344; P. C. McGuire and P. J. Steinhardt, arXiv:astro-ph/0105567; G. Zaharijas and G. R. Farrar, Phys. Rev. D 72, 083502 (2005)

19. M. Y. Khlopov, arXiv:0801.0167 [astro-ph]; M. Y. Khlopov, arXiv:0801.0169 [astro-ph].

20. M. Yu. Khlopov, A. G. Mayorov, E.Yu. Soldatov, Bled Workshops in Physics 10, N-2 (2009)to be published; arXiv:0911.5606 [astro-ph].

21. M. Y. Khlopov and C. Kouvaris, Phys. Rev. D 78, 065040 (2008)

22. R. Bernabei et al., Rivista Nuovo Cimento 26, 1 (2003)

23. R. Bernabei et al. [DAMA Collaboration], Eur.Phys.J C56, 333 (2008)

24. R. N. Cahn and S. L. Glashow, Science 213, 607 (1981); M. Pospelov, Phys. Rev. Lett. 98, 231301 (2007); K. Kohri and F. Takayama, Phys. Rev. D 76, 063507 (2007).

25. A. G. Mayorov, "Search for Stable heavy charged particles of dark matter in the experiment PAMELA", PreDiplom Report, MIFI, Moscow, 2008.

26. D. P. Finkbeiner and N. Weiner, Phys. Rev. D 76, 083519 (2007)

27. B. J. Teegarden et al, Astrophys. J. 621, 296 (2005)

28. D. McCammon et al, Nucl. Instrum. Methods A 370, 266 (1996); D. McCammon et al, Astrophys. J. 576, 188 (2002).

29. K. Belotsky et al, arXiv:astro-ph/0606350.

30. D. S. Akerib et al. [CDMS Collaboration], Phys. Rev. Lett. 96, 011302 (2006); Z. Ahmed et al. [CDMS Collaboration], arXiv:0802.3530 [astro-ph].

31. M. Yu. Khlopov, A. G. Mayorov, E.Yu. Soldatov, Bled Workshops in Physics 9, 24 (2008)

32. L. D. Landau, E. M. Lifshitz Quantum Mechanics: Non-Relativistic Theory (Fizmatlit, Moscow, 2004).

33. O. Kamaev for the CDMS Collaboration, arXiv:0910.3005 [hep-ex].

34. F. Petriello and K. M. Zurek, JHEP 0809, 047 (2008); R. Foot, Phys. Rev. D 78, 043529 (2008); J. L. Feng, J. Kumar and L. E. Strigari, arXiv:0806.3746 [hep-ph]; J. L. Feng, J. Kumar, J. Learned and L. E. Strigari, arXiv:0808.4151 [hep-ph]; E. M. Drobyshevski, arXiv:0811.0151 [astro-ph].

35. G. B. Gelmini, arXiv:0810.3733 [hep-ph]. 